\title{
Is the result of modified Allen's test still accurate after endoscopic thoracic sympathectomy?
}

\author{
Jiaheng Zhang ${ }^{1 *}$, Yuanjun Cheng ${ }^{2 \#}$, Donglai Chen ${ }^{3 *}$, Fuquan Zhang', Shanzhou Duan ${ }^{1}$, Lei Chen ${ }^{1}$, \\ Chang Chen ${ }^{3}$, Yonghua Sang ${ }^{1}$, Li Shi $^{1}$, Wentao Yang ${ }^{1 *}$, Yongbing Chen ${ }^{1 *}$
}

${ }^{1}$ Department of Thoracic Surgery, the Second Affiliated Hospital of Soochow University, Suzhou 215004, China; ${ }^{2}$ Department of Cardiothoracic Surgery, People's Hospital of Chizhou, Chizhou 247100, China; ${ }^{3}$ Department of Thoracic Surgery, Shanghai Pulmonary Hospital, Tongji University, School of Medicine, Shanghai 200433, China

Contributions: (I) Conception and design: J Zhang, Y Cheng; (II) Administrative support: Y Chen, W Yang; (III) Provision of study materials or patients: Y Chen, W Yang; (IV) Collection and assembly of data: F Zhang, S Duan, L Chen; (V) Data analysis and interpretation: C Chen, Y Sang, L Shi; (VI) Manuscript writing: All authors; (VII) Final approval of manuscript: All authors.

\#These authors contributed equally to this work.

*These authors are both listed as senior authors.

Correspondence to: Yongbing Chen, MD, PhD. Department of Thoracic Surgery, the Second Affiliated Hospital of Soochow University, 1055 Sanxiang Road, Gusu District, Suzhou 215004, China. Email: chentongt@sina.com.

Background: The radial artery (RA) is increasingly being used for coronary artery bypass grafting (CABG). Endoscopic thoracic sympathectomy (ETS) has been shown to block innervation of sympathetic nerves of upper limbs, which reduces sweating of hands and dilates blood vessels. The modified Allen's test (MAT) is one of the commonest methods of assessing collateral arm flow prior to RA harvest, though it has limitations. However, the reliability of MAT after ETS remains unclear. We therefore investigated the effects of ETS on the results of MAT.

Methods: A retrospective cohort study was conducted on 164 consecutive Chinese patients with palmar hyperhidrosis who underwent ETS between January 2016 and January 2019. The medical records were reviewed concerning the ultrasound examination and MAT results of their RAs and ulnar arteries (UAs) in both forearms before and after ETS.

Results: The performance of ETS significantly increased the diameter of the right RA from $2.731 \pm 0.122$ to $3.102 \pm 0.114 \mathrm{~mm}$ in men and from $2.347 \pm 0.074$ to $2.915 \pm 0.162 \mathrm{~mm}$ in women. Similar effects of ETS were observed in expanding the diameters of the left RA and the UA. Meanwhile, there was no significant effect of ETS on systolic blood pressure (BP) and heart rate (HR). Overall, retesting of patients following ETS with a preoperative positive MAT result revealed a transition to a negative result.

Conclusions: ETS was effective in dilating RA in both men and women, which could lead to a false negative preoperative MAT result. Patients should be questioned about their history of ETS if their RAs are to be harvested for CABG. More studies are warranted to evaluate the safety of RA as a coronary artery graft after ETS.

Keywords: Endoscopic thoracic sympathectomy (ETS); modified Allen's test (MAT); radial artery (RA); coronary artery bypass grafting $(\mathrm{CABG})$; palmar hyperhidrosis

Submitted Oct 08, 2019. Accepted for publication Dec 10, 2019.

doi: $10.21037 /$ jtd.2019.12.112

View this article at: http://dx.doi.org/10.21037/jtd.2019.12.112 


\section{Introduction}

The radial artery (RA) has been used for coronary artery bypass grafting (CABG) since the 1970s (1). However, the use of the RA as a coronary artery graft was soon abandoned because of poor patency rates, which could have resulted from arterial spasm. In the meantime, most patients who underwent $\mathrm{CABG}$, received saphenous vein (SV) grafts in addition to internal thoracic artery (ITA) grafts for replacing the left anterior descending coronary artery (2). With the intervention of drugs and the improvement in surgical skills, revival of RA grafts ensued two decades later, because it had been found that the long-term patency rates of RA grafts were significantly higher than previously reported (1). Nowadays, it has been reported that the patency rate has reached nearly $90 \%$ in patients who accepted RA grafts (3).

With increasing use of endoscopes and ultrasonic knifes, especially in endoscopic RA harvesting, the harvest of the RA conduit has become more convenient than that of the internal mammary artery (4). Moreover, the RA conduit is associated with a lower postoperative complication rate, which reveals its superiority over SV as a graft (5). In addition to paresthesia at the operated side (6) and arteriosclerosis of the RA (7), the major problem is that the harvested RA is much easier to twitch than the SV and the ITA.

Palmar hyperhidrosis is characterized by abnormal hypersecretion of sweat glands in the hands, which is associated with hyperexcitability of ipsilateral sympathetic nerves. Endoscopic thoracic sympathectomy (ETS) can block the sympathetic innervation of the sweat glands of the upper limbs by cutting off the sympathetic nerve chain and its lateral branches, leading to dilation of blood vessels and improved hemodynamics eventually.

The modified Allen's test (MAT) is the commonest method of assessing collateral arm flow prior to RA harvest, though it has limitations (8). Winkler et al. (9) reported that surgeons were least keen to proceed to RA harvest if the Allen's test was positive ( $>10$ seconds). Incomplete surgical sympathectomy, reinnervation of the sympathetic pathways, or hypersensitivity of the noradrenergic receptors regulating the precapillary sphincters reacting to very small amounts of circulating catecholamines have all been implicated as factors leading to recurrence of forearm vasospasm $(10,11)$. We assumed that the changes in forearm arteries after ETS may lead to a false negative MAT result. Thus, the MAT result is of significance before the harvest of RA.

To study the effects of ETS on the hemodynamics of forearm arteries and MAT results, we performed ultrasonic tests to monitor the preoperative and postoperative hemodynamic changes, which could provide clinical evidence and theoretical analysis for the harvest of RAs for patients in need of CABG.

\section{Methods}

We consecutively reviewed the medical records of 164 Chinese patients with palmar hyperhidrosis from January 2016 to January 2019 in this retrospective cohort study. We performed ultrasound measurement and MAT of their RAs and ulnar arteries (UAs) in both forearms before and after ETS. This study was approved by the Institutional Review Board of the Second Affiliated Hospital of Soochow University. Due to the retrospective nature of this study, informed consent of patients was waived.

\section{Study population}

All included patients met the diagnostic criteria established by Hornberger et al. (12) in 2004, which were as follows: the local hypersecretion of sweat glands visible to the naked eye without obvious inducement lasting for more than 6 months, excluding secondary factors (such as drugs, endocrine diseases, neurological diseases, etc.). Meanwhile, at least two of the following criteria should be met: (I) symmetrical sweating area bilaterally; (II) affects daily work and life; (III) attacks at least once a week; (IV) the age of onset is less than 25 years; (V) a positive family history; and (VI) local sweating stops during sleep.

\section{Surgical approach: ETS}

We have been performing this surgery for over a decade and all of these cases were performed by the same experienced team. During the operation, T3 and T4 sympathectomy was performed along the sympathetic chain using a coagulation hook at the caput costae bilaterally. A 2-cm extension around the sympathetic chain was isolated and cauterized to ensure that the trunk and lateral branches of the nerve could be cut off completely. Hand temperature monitoring was done routinely.

After a successful operation, the incision was sutured intermittently, and a tube was placed into the chest cavity with its distal end placed under water. A positive pressure ventilation was conducted for a few seconds after lung re-expansion, then the tube was removed after the 
exhaustion of remnant air in the chest cavity. The incision was subsequently closed and covered without placing an intrathoracic drainage tube.

\section{Ultrasonography study of forearm arteries}

The ultrasonography studies were performed 1 day before ETS, and subsequently 1 day, 1 month, and 6 months after ETS. All of these ultrasonography studies were performed by the same sonographer. Two-dimensional vascular images and color Doppler ultrasonography of both the right and left forearm arteries were performed by an experienced ultrasonologist using a SonoSite TITAN portable ultrasound system (SonoSite Inc., Bothell, WA, USA). The transducer was placed perpendicular to the arterial wall to acquire an optimal image of the vessel. Once in position, the site was marked so that the same segment of the vessel was imaged throughout the examinations. The image was recorded in Audio Video Interleaved (AVI) format that was transferred to an external computer for subsequent analysis.

Patients lay in a supine position with both arms comfortably abducted and the wrist supported in a temperature-controlled operation room. The preoperative inner diameters of lumina of RA and UA on both sides were measured immediately after the respective styloid processes in each arm. Postoperative measurement of the bilateral RA and UA diameters was repeated after performing ETS. The mean inner diameter was defined as an average value of three perpendicular readings.

\section{MAT}

The same physician performed MATs synchronously with the ultrasonography at four corresponding time points. The MAT was performed by simultaneous compression of both the RA and UA. A cutoff point of 10 seconds was used (13). The patient was asked to make a fist and open his/her hand several times until the palm turned pale. The compression on the UA was then released. The MAT was defined as negative if full blushing of the palm occurred within 10 seconds, otherwise the MAT was defined as positive. For patients with a positive MAT result, the test was repeated after performance of ETS.

\section{Data collection}

In addition to bilateral arterial diameters, patients' characteristics and wrist circumference, MAT results and blood pressure (BP) were recorded before and after ETS. Wrist circumference was measured 2-cm above the tip of the radial styloid process.

\section{Statistical analysis}

Statistical analysis was performed using IBM SPSS Statistics 19.0 (IBM Corp., Armonk, New York, USA). Categorical data were presented as absolute values and percentages, whereas continuous data were presented as mean \pm standard deviation. The nature of distribution of the data was determined using the one-sample Kolmogorov-Smirnov test. Student's $t$-test and Mann-Whitney $\mathrm{U}$ test were used to compare continuous data, as appropriate. Categorical data were compared using the chi-square test with the appropriate degrees of freedom. Statistical significance was set at $\mathrm{P}<0.05$.

\section{Results}

\section{Clinical characteristics of patients}

A total of 164 patients with palmar hyperhidrosis were included: $74(45.1 \%)$ were male and 90 (54.9\%) were female. Baseline characteristics categorized by gender are detailed in Table 1 . The male patients were significantly taller $(\mathrm{P}<0.0001)$ and heavier $(\mathrm{P}<0.0001)$ and had a greater wrist circumference $(\mathrm{P}<0.0001)$ than the female patients. Moreover, the RA was significantly smaller in women than men $(\mathrm{P}<0.0001)$ and the right $\mathrm{RA}$ was the largest. Several factors, such as smaller RA diameter, female sex, younger age, lower body mass index, diabetes, and active smoking have been identified as predisposing factors that increase the risk of developing RA spasm.

\section{Effects of ETS on the MAT result}

Figure 1 shows the percentage of patients with a negative MAT result, both preoperatively and postoperatively. The MAT was observed as negative in a large proportion of patients before ETS, and the negative rate of the MAT was observed as different on both sides. The negative rate of the left hand was higher in male patients than that in female patients, whereas that of the right hand was just the reverse. After accepting ETS, all patients with a positive MAT result altered to show a negative result within 6 months. 
Table 1 Patients' characteristics grouped by sex

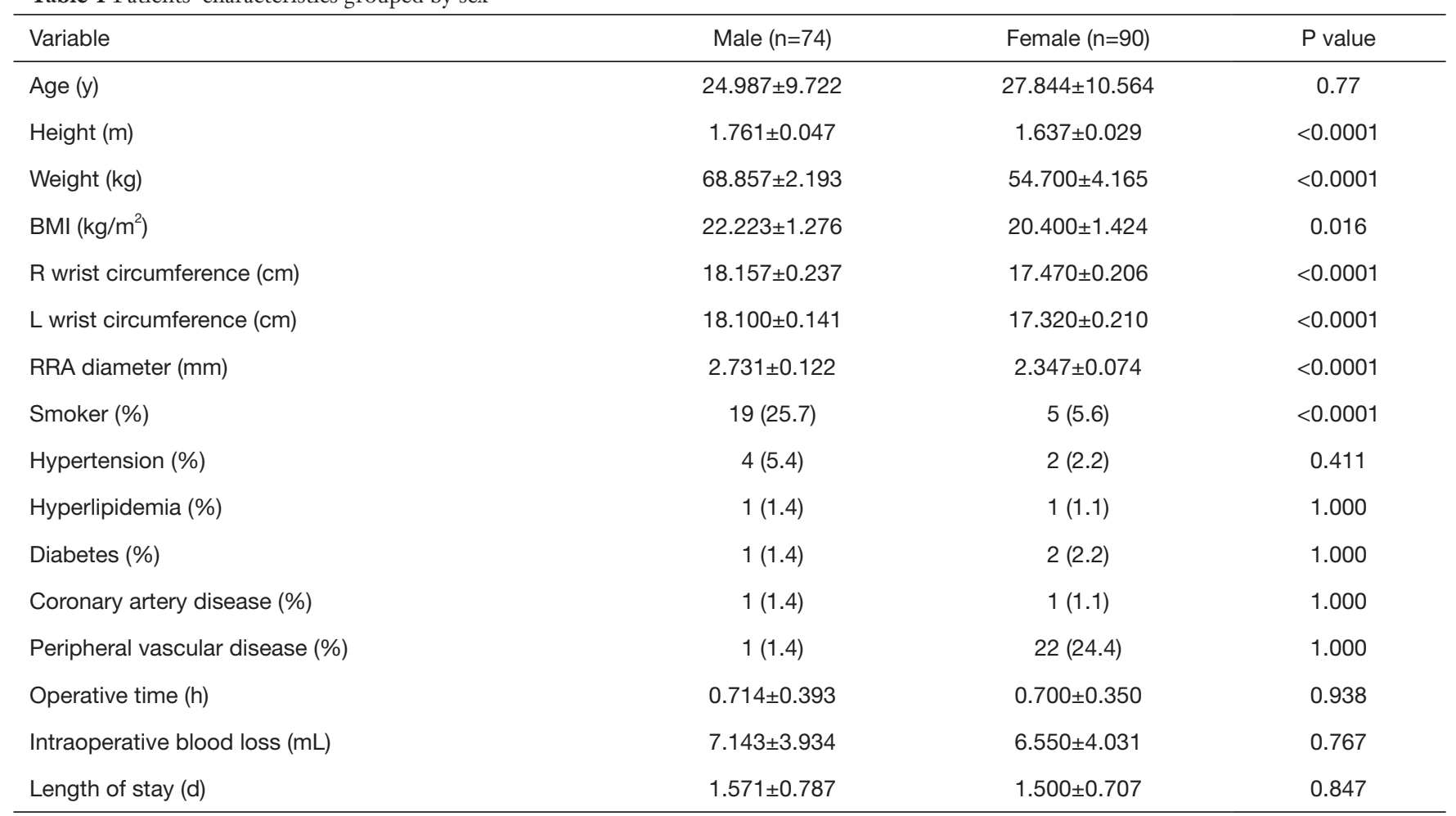

Data are shown as mean \pm standard deviation. BMI, body mass index; RRA, right radial artery.

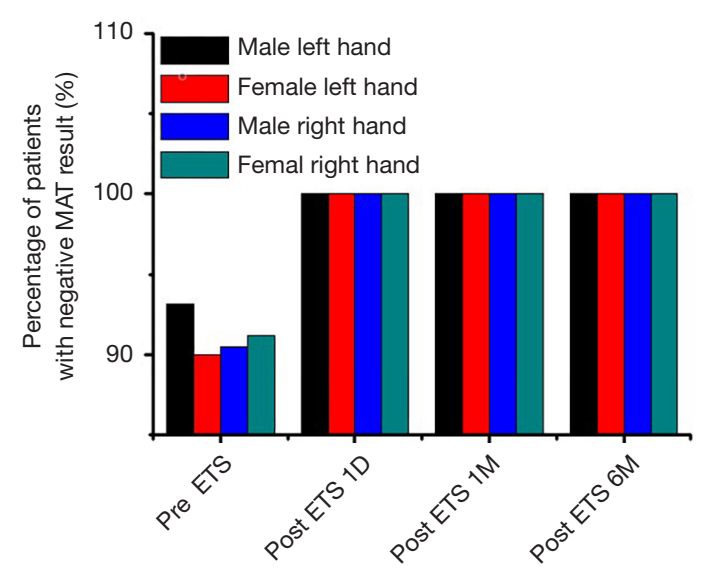

Figure 1 The percentage of patients with a negative MAT result preoperatively and postoperatively. MAT, modified Allen's test; ETS, endoscopic thoracic sympathectomy.

\section{Effect of ETS on diameters and maximal blood velocity} $\left(V_{\text {max }}\right)$ of forearm arteries

The diameters of the RA and UA on both sides are listed in Figures 2 and 3. The male patients had significantly larger wrist arteries than the female patients $(\mathrm{P}<0.05)$. For both sexes, the RA at the wrist was thicker than the ipsilateral $\mathrm{UA}$, and the right RA had the largest mean diameter. ETS significantly increased the diameters of the RA and UA of the patient cohort $(\mathrm{P}<0.05)$. The maximal blood velocity $\left(\mathrm{V}_{\text {max }}\right)$ of the forearm arteries are shown in Figures 4 and 5. ETS significantly increased the $V_{\max }$ of the RA and UA in both genders.

\section{Effect of ETS on systolic BP and beart rate (HR)}

The BP and HR of both sexes changed little within 

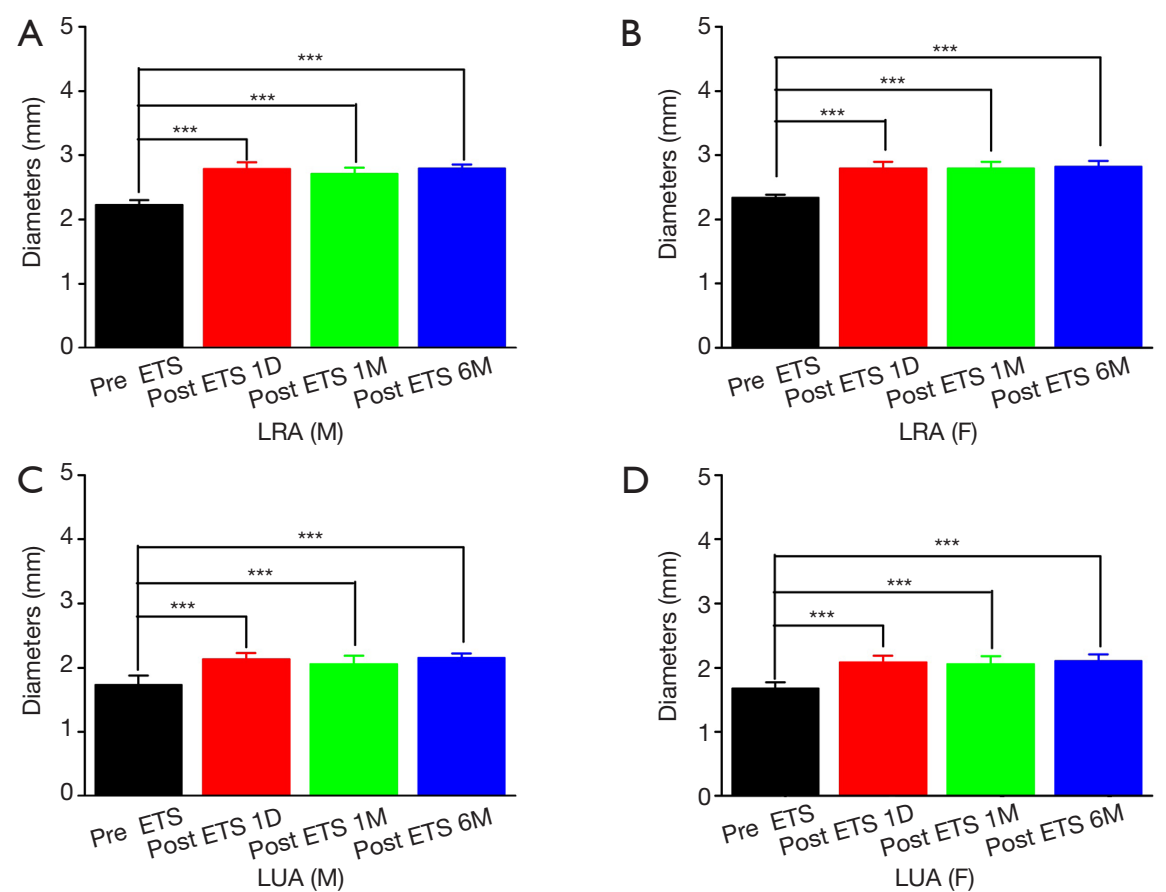

LUA (F)

Figure 2 The changes in diameters of the left radial artery (LRA) and the left ulnar artery (LUA) in males and females. (A) The changes in diameters of the LRA in males; (B) the changes in diameters of the LRA in females; (C) the changes in diameters of the LUA in males; (D) the changes in diameters of the LUA in females. ${ }^{* *}, \mathrm{P}<0.0001$.
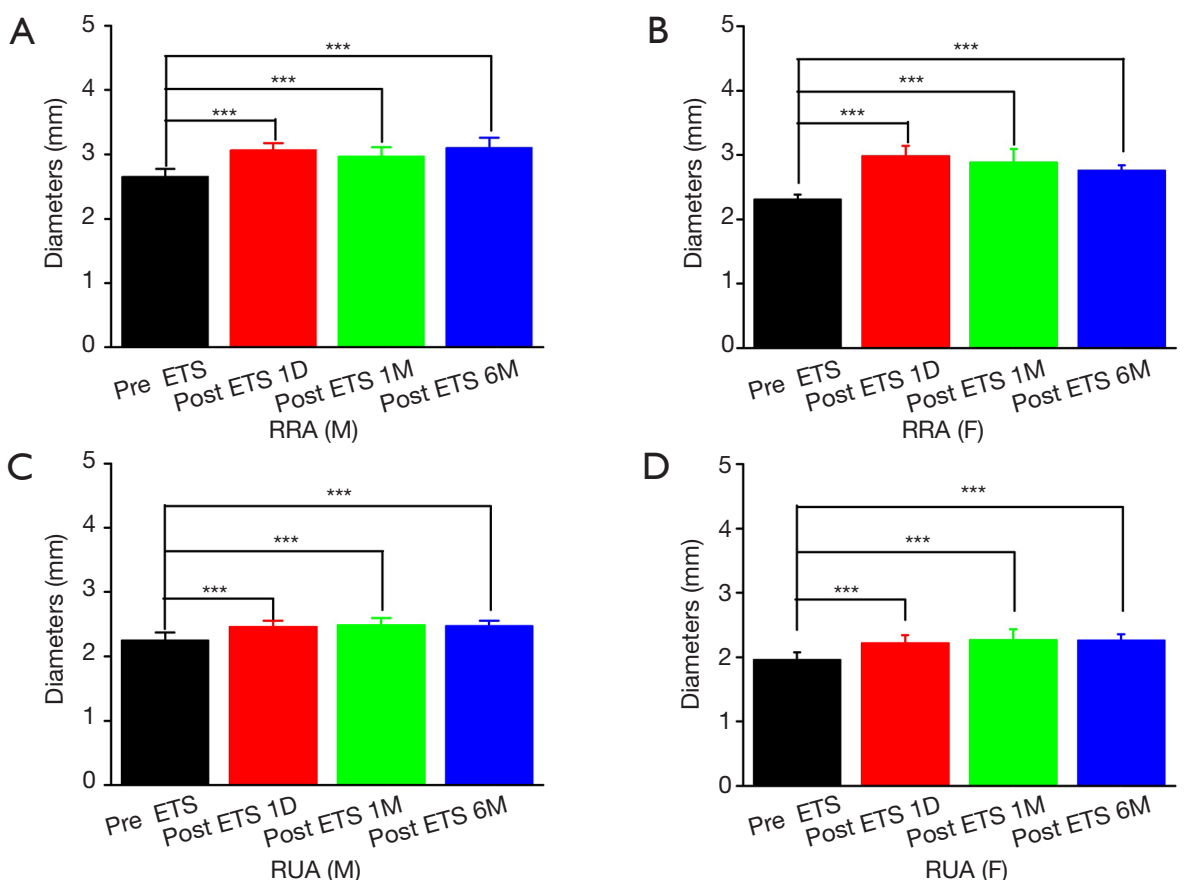

Figure 3 The changes in diameters of the right radial artery (RRA) and the right ulnar artery (RUA) in males and females. (A) The changes in diameters of the RRA in males; (B) the changes in diameters of the RRA in females; (C) the changes in diameters of the RUA in males; (D) the changes in diameters of the RUA in females. ${ }^{* *}, \mathrm{P}<0.0001$. 

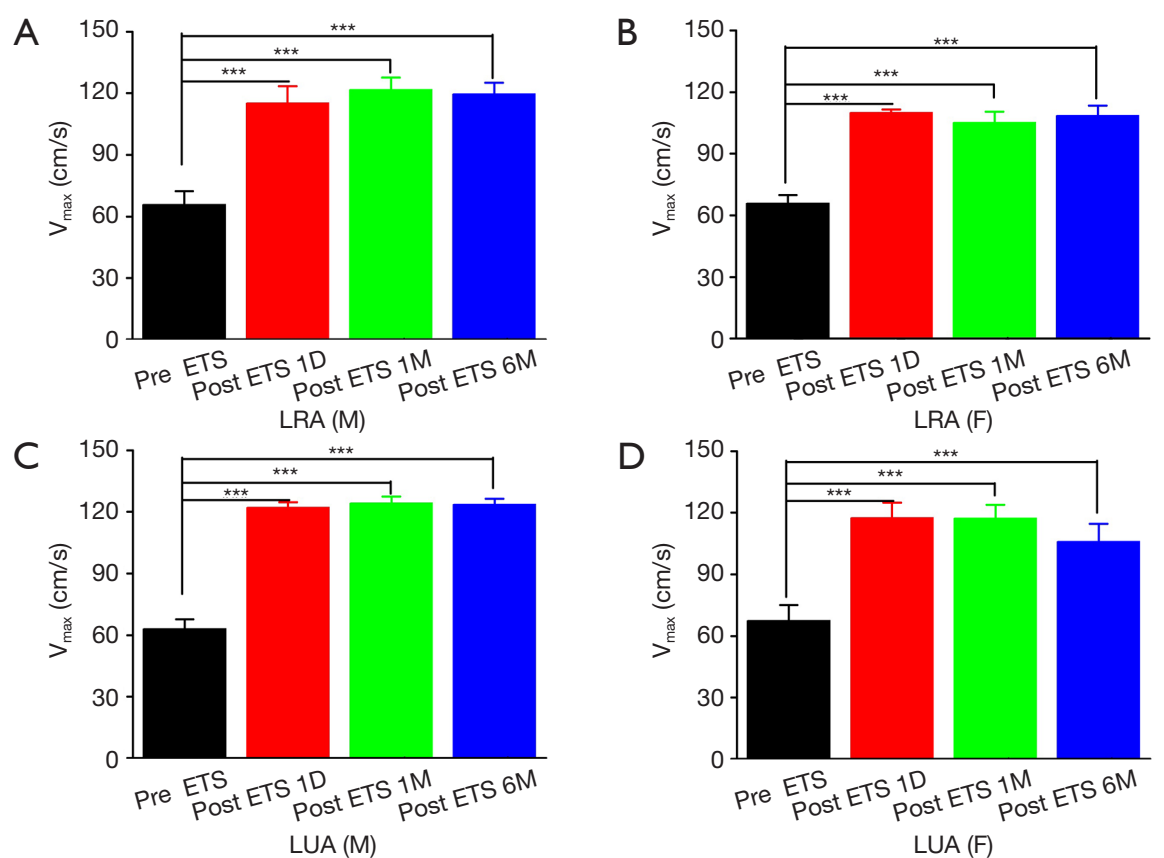

Figure 4 The changes in the maximal blood velocity of the left radial artery (LRA) and the left ulnar artery (LUA) in males and females. (A) The changes in the maximal blood velocity of the LRA in males; (B) the changes in the maximal blood velocity of the LRA in females; (C) the changes in the maximal blood velocity of the LUA in males; (D) the changes in the maximal blood velocity of the LUA in females. ***, $\mathrm{P}<0.0001$.
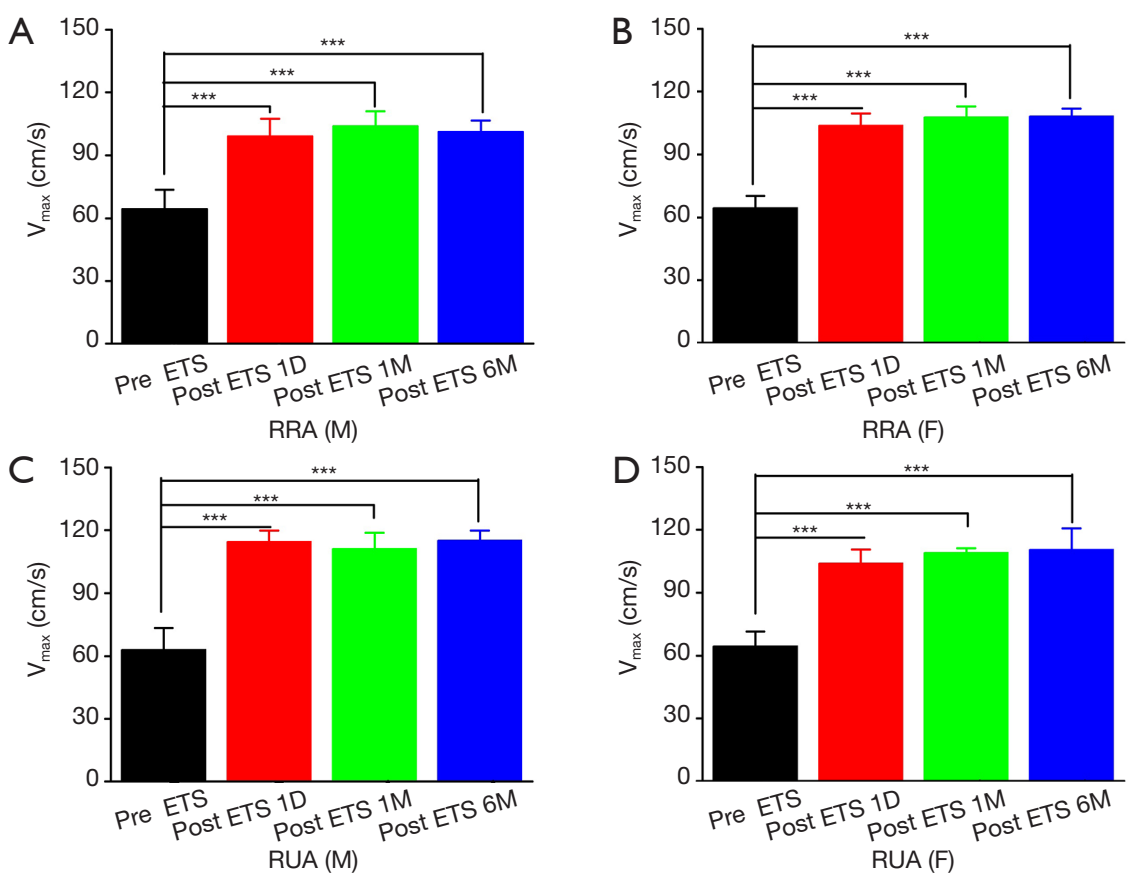

Figure 5 The changes in the maximal blood velocity of the right radial artery (RRA) and the right ulnar artery (RUA) in males and females. (A) The changes in the maximal blood velocity of the RRA in males; (B) the changes in the maximal blood velocity of the RRA in females; (C) the changes in the maximal blood velocity of the RUA in males; (D) the changes in the maximal blood velocity of the RUA in females. ${ }^{* * *}$, $\mathrm{P}<0.0001$. 

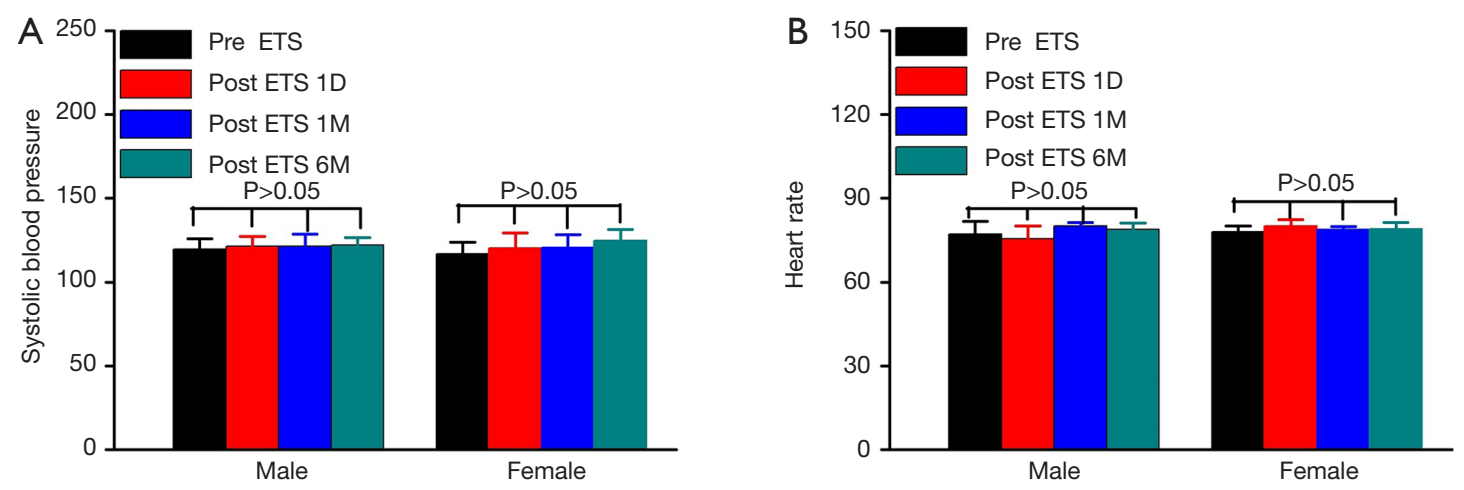

Figure 6 The changes in blood pressure and heart rate in males and females. (A) The changes in blood pressure in males and females; (B) the changes in heart rate in males and females.

6 months after ETS (Figure 6). Therefore, ETS was welltolerated and exhibited no significant impact on BP and HR of our patient cohort.

\section{Discussion}

A recent study has reported that the reliability of MAT is limited (14). However, most physicians still consider MAT as an essential pre-operative procedure. The mean diameter of RA varies among countries, such as $2.4 \pm 0.4 \mathrm{~mm}$ in Japan (15), $2.6 \pm 0.4 \mathrm{~mm}$ in Korea (16), and 2.38 $\pm 0.56 \mathrm{~mm}$ in China (17). Our study examined forearm arterial diameters and the effect of ETS on RA using high resolution ultrasound; the results were refined and categorized mainly by gender and laterality.

The incidence of coronary heart disease is increasing with each passing year, and the death rate has far exceeded that due to cancer; hence coronary heart disease poses a great threat to human health. According to the American College of Cardiology (ACC)/American Heart Association (AHA) guidelines, patients with coronary heart disease may be suitable for surgical treatment when drug treatment and internal interventional therapy are ineffective or of great risk. In addition, patients whose vessels are bent, completely occluded, or bifurcated with narrow left main coronary artery, are not suitable candidates for interventional therapy, either for balloon dilation or for the use of coated stents. Surgical revascularization has been considered the best solution because the bridging materials used in CABG are autogenous vessels. The long-term patency rate of coronary artery and vein was higher than that of most advanced coronary stents. Even ten years after the operation, more than $90 \%$ of the bridging vessels remain open. According to mid- and long-term follow-up results, the patency rate of RA is significantly better than SV (18-20). Regardless of age, sex, diabetes status, and left ventricular function, the use of RA is associated with a significant reduction in mortality during follow-up without an increase in perioperative mortality or morbidity (21). The easy accessibility, convenience in simultaneous harvest with other arterial conduits, and the potentially optimal caliber and length make the RA an almost ideal conduit for CABG (21).

To serve as a bridging vessel, the RA needs to be examined by MAT before CABG. The safety assessment of the harvest includes the following algorithm of tests: ultrasound, the Allen's test, and pulse oximetry (22). The quality criteria that are used to exclude a RA from harvesting are small size of the artery, diffuse atherosclerosis, calcifications, and severe neointimal hyperplasia (22). However, there may be more false results, either positive or negative, than expected, which depend on the subjectivity of different examiners. If the Allen's test is negative, it is safe to harvest the RA $(8,13)$. If it is positive, further examinations are needed to ensure adequate blood supply in the ipsilateral UA after harvest of the RA $(8,13)$.

ETS may treat primary hyperhidrosis by cutting off the corresponding thoracic sympathetic nerve chain and lateral branches, so that the sympathetic innervation in the sweat glands of the upper limbs is no longer effective. Notably, it is speculated that hemodynamics of upper limbs may also be affected. Schick et al. (23) found that the blood flow in the RA and UA in patients with primary hyperhidrosis increased significantly 2 days after ETS. In this study, the diameter and blood flow velocity of the RA and UA increased significantly after ETS, which was maintained for at least 6 months. We speculate that this was due to the loss of sympathetic dominance. Meanwhile, no abnormal changes in BP and HR were observed in post-ETS patients, 
which revealed that ETS had little impact on cardiac functions or hemodynamics.

RA spasm is one of the most common complications encountered during transradial procedures. Spasmogens in arterial grafts are well studied and include endotheliumderived contracting factors (e.g., endothelin-1), prostaglandins (e.g., thromboxane A2 or its mimetic PGF2 $\alpha$ ), $\alpha$-adrenoceptor agonists (e.g., norepinephrine, methoxamine, and phenylephrine), platelet-derived substances (e.g., 5 -hydroxytryptamine), and the cellular membrane-depolarizing agent potassium, as well as mast cells and histamine (24). Thus, the changes we observed in the RA were not sufficient to assess the safety of RA as a graft. Choudhary et al. have reported that diabetes mellitus, which causes generalized endothelial dysfunction, increased the risk of RA spasm or the vasomotor responses of RA grafts to physiological vasoconstrictors such as adrenalin, PGF2 $\alpha$, and angiotensin II (25). Moreover, smoking habit is also a pre-procedurally known factor that can predict the occurrence of RA spasm (26). To the best of our knowledge, there is no evidence available concerning the surgical treatment for RA spasm.

Removal of the RA has the potential to reduce forearm blood supply, leaving it completely dependent on the collateral circulation from the UA and can theoretically expose the patient to the risk of forearm ischemia. Therefore, it is worth taking the MAT result into account. In our cohort, 29 patients with a positive MAT result showed a negative result after ETS, which indicated that ETS increased the inner diameter and blood flow of both the RA and the UA.

Our study had several limitations. Due to the retrospective nature of our single-center study, selection and performance biases were inevitable. Although we have demonstrated that ETS had a significant impact on the diameter of the RA in patients with palmar hyperhidrosis, we were unable to monitor the long-term changes in the diameter of the RA in these patients due to a limited follow-up period. In addition, there is no denying the fact that other factors (mood, temperature, pain) associated with the RA might have been omitted in our study, which could have affected the patency rate of the RA. Notably, it is our speculation that long-term loss of epinephrine stimulation on the RA after ETS would decrease the activity of epinephrine receptors on the surface of the blood vessel, which might lower the likelihood of RA spasm. More studies and long-term followup are needed to address these limitations.

\section{Conclusions}

In patients with palmar hyperhidrosis, ETS was effective in dilating the RA in both men and women, which can lead to a false negative MAT result. Patients should be inquired about history of ETS if their RAs are supposed to be harvested for CABG. More studies are warranted to evaluate the safety of using the RA as a coronary artery graft after ETS.

\section{Acknowledgments}

We thank International Science Editing (http://www. internationalscienceediting.com) for editing this manuscript. Funding: Supported by the projects from Shanghai Hospital Development Center (SHDC12015116), the National Natural Science Foundation of China (NSFC81770091), Clinical Research Foundation of Shanghai Pulmonary Hospital (FK1943, FK1936, FK1942, FK1941), Shanghai Municipal Health Commission (2018ZHYL0102, 2019SY072, 201940018), Science and Technology Commission of Shanghai Municipality (15411968400 and 14411962600), Suzhou Key Laboratory of Thoracic Oncology (SZS201907), Suzhou Key Discipline for Medicine (SZXK201803), the Science and Technology Research Foundation of Suzhou Municipality (SYS2018063, SYS2018064) and Municipal Program of People's Livelihood Science and Technology in Suzhou (SS2019061).

\section{Footnote}

Conflicts of Interest: The authors have no conflicts of interest to declare.

Ethical Statement: The authors are accountable for all aspects of the work in ensuring that questions related to the accuracy or integrity of any part of the work are appropriately investigated and resolved. This study was approved by the Institutional Review Board of the Second Affiliated Hospital of Soochow University. Due to the retrospective nature of this study, informed consent of patients was waived.

Open Access Statement: This is an Open Access article distributed in accordance with the Creative Commons Attribution-NonCommercial-NoDerivs 4.0 International License (CC BY-NC-ND 4.0), which permits the noncommercial replication and distribution of the article with the strict proviso that no changes or edits are made and the original work is properly cited (including links to both the formal publication through the relevant DOI and the license). See: https://creativecommons.org/licenses/by-nc-nd/4.0/. 


\section{References}

1. Acar C, Jebara VA, Portoghese M, et al. Revival of the radial artery for coronary artery bypass grafting. Ann Thorac Surg 1992;54:652-9.

2. Elbardissi AW, Aranki SF, Sheng S, et al. Trends in isolated coronary artery bypass grafting: An analysis of the Society of Thoracic Surgeons adult cardiac surgery database. J Thorac Cardiovasc Surg 2012;143:273-81.

3. Ji Q, Song K, Shen J, et al. Long-term patency rate of radial artery conduits in Chinese patients undergoing off-pump coronary artery bypass grafting. Int Heart J 2019;60:1276-83.

4. Kobayashi J. Radial artery as a graft for coronary artery bypass grafting. Circ J 2009;73:1178-83.

5. Cohen G, Tamariz MG, Sever JY, et al. The radial artery versus the saphenous vein graft in contemporary CABG: A case-matched study. Ann Thorac Surg 2001;71:180-6.

6. Siminelakis S, Karfis E, Anagnostopoulos C, et al. Harvesting radial artery and neurologic complications. J Card Surg 2004;19:505-10.

7. Chen XJ, Chen X, Xie D, et al. Radial artery as conduit is safe and effective in coronary bypass surgery in the elderly single-central results from 326 patients. Circ J 2009;73:1049-54.

8. Ronald A, Patel A, Dunning J. Is the Allen's test adequate to safely confirm that a radial artery may be harvested for coronary arterial bypass grafting? Interact Cardiovasc Thorac Surg 2005;4:332-40.

9. Winkler J, Lohr J, Bukhari RH, et al. Evaluation of the radial artery for use in coronary artery bypass grafting. $\mathrm{J}$ Vasc Technol 1998;22:23-9.

10. Claes G. Indications for endoscopic thoracic sympathectomy. Clin Auton Res 2003;13:I16e9.

11. Matsumoto Y, Ueyama T, Endo M, et al. Endoscopic thoracic sympathicotomy for Raynaud's phenomenon. J Vasc Surg 2002;36:57e61.

12. Hornberger J, Grimes K, Naumann M, et al. Recognition, diagnosis, and treatment of primary focal hyperhidrosis. J Am Acad Dermatol 2004;51:286.

13. Kohonen M, Teerenhovi $\mathrm{O}$, Terho T, et al. Is the Allen test reliable enough? Eur J Cardiothorac Surg 2007;32:902-5.

14. Romeu-Bordas Ó, Ballesteros-Peña S. Reliability and validity of the modified Allen test: A systematic review and metanalysis. Emergencias 2017;29:126-35.

15. Nagai S, Abe S, Sato T, et al. Ultrasonic assessment of vascular complications in coronary angiography and angioplasty after transradial approach. Am J Cardiol
1999;83:180-6.

16. Yoo B S, Yoon J, Ko J Y, et al. Anatomical consideration of the radial artery for transradial coronary procedures: arterial diameter, branching anomaly and vessel tortuosity. Int J Cardiol 2005;101:427.

17. Yan ZX, Zhou YJ, Zhao YX, et al. Anatomical study of forearm arteries with ultrasound for percutaneous coronary procedures. Circ J 2010;74:686-92.

18. Benedetto U, Raja SG, Albanese A, et al. Searching for the second best graft for coronary artery bypass surgery: A network meta-analysis of randomized controlled trials. Eur J Cardiothorac Surg 2015;47:59-65; discussion 65.

19. Collins P, Webb CM, Chong CF, et al. Radial artery versus saphenous vein patency randomized trial: Five-year angiographic follow-up. Circulation 2008;117:2859-64.

20. Deb S, Cohen EA, Singh SK, et al. Radial artery and saphenous vein patency more than 5 years after coronary artery bypass surgery: Results from RAPS (Radial Artery Patency Study). J Am Coll Cardiol 2012;60:28-35.

21. Gaudino M, Rahouma M, Abouarab A, et al. Radial artery versus saphenous vein as the second conduit for coronary artery bypass surgery: A meta-analysis. J Thorac Cardiovasc Surg 2019;157:1819-1825.e10.

22. Vukovic P, Peric M, Radak S, et al. Preoperative insight into the quality of radial artery grafts. Angiology 2017;68:790-4.

23. Schick CH, Fronek K, Held A, et al. Differential effects of surgical sympathetic block on sudomotor and vasoconstrictor function. Neurology 2003;60:1770-6.

24. He GW, Taggart DP. Spasm in arterial grafts in coronary artery bypass grafting Surgery. Ann Thorac Surg 2016;101:1222-9.

25. Choudhary BP, Antoniades C, Brading AF, et al. Diabetes mellitus as a predictor for radial artery vasoreactivity in patients undergoing coronary artery bypass grafting. J Am Coll Cardiol 2007;50:1047-53.

26. Giannopoulos G, Raisakis K, Synetos A, et al. A predictive score of radial artery spasm in patients undergoing transradial percutaneous coronary intervention. Int J Cardiol 2015;188:76-80.

Cite this article as: Zhang J, Cheng Y, Chen D, Zhang F, Duan S, Chen L, Chen C, Sang Y, Shi L, Yang W, Chen Y. Is the result of modified Allen's test still accurate after endoscopic thoracic sympathectomy? J Thorac Dis 2020;12(3):696-704. doi: 10.21037/jtd.2019.12.112 